\title{
Open Source Implementation of Different Variants of Jiles-Atherton Model of Magnetic Hysteresis Loops
}

\author{
R. SZEWCZYK ${ }^{a, *}$ AND P. CHENG ${ }^{b}$ \\ ${ }^{a}$ Institute of Metrology and Biomedical Engineering, Warsaw University of Technology, \\ sw. A Boboli 8, 02-525 Warsaw, Poland \\ ${ }^{b}$ Department of Electronics Design, Mid Sweden University, SE-851 70 Sundsvall, Sweden
}

\begin{abstract}
Jiles-Atherton model is one of the most advanced and most popular models of magnetic hysteresis loop. However, this model is considering different physical phenomena and computational issues. As a result, cross-validation of the results of modelling performed by different authors became difficult. For this reason, the open-source MATLAB/OCTAVE based implementation of Jiles-Atherton model was developed. Proposed implementation covers isotropic model of magnetic hysteresis loops as well as uniaxial and grain oriented electrical steel anisotropy. Moreover, the corrections proposed by Venkataraman together with different approaches to derivative of the anhysteretic magnetization are considered. Developed library is freely available together with the examples of magnetic hysteresis loops. As a result, it can be the base for further development of Jiles-Atherton model for better understanding of magnetization process as well as modelling the inductive components.
\end{abstract}

DOI: 10.12693/APhysPolA.133.654

PACS/topics: 75.50.Bb, 75.50.Gg, 75.60.Ej

\section{Introduction}

Jiles-Atherton model is one of the most popular models of magnetic hysteresis loop. Since its introduction [1] in 1984 this model was developed and updated considering different physical phenomena, such as uniaxial or cubic anisotropy of magnetic material $[2,3]$, dynamic magnetization [4], as well as advanced methods of determination of model parameters [5-7]. Moreover, different computational issues connected with Jiles-Atherton model were considered [8], mostly caused by solving of differential equations stating the model. As a result, cross-validation of the results of modelling performed by different authors became difficult.

To overcome this problem, open-source implementation of Jiles-Atherton model was developed. This implementation is based on open-source OCTAVE software, however, it can be also used with MATLAB. Software is freely available together with the source code at github.com/romanszewczyk/JAmodel/tree/master/ 10_APPA.

\section{Jiles-Atherton model}

In the Jiles-Atherton model, the effective magnetizing field $H_{e}$ is given as [1]:

$$
H_{e}=H+\alpha M,
$$

where $H$ is the magnetizing field strength, $M$ is magnetization of the material and $\alpha$ is inter-domain coupling according to the Bloch model.

The principal concept in the Jiles-Atherton model is the idea of anhysteretic magnetization [1]. Such magne-

*corresponding author; e-mail: szewczyk@mchtr.pw.edu.pl tization can be observed experimentally during the demagnetization of material in the presence of offset magnetizing field [9]. However, the experimental setup for such experiment is quite sophisticated.

Anhysteretic magnetization $M_{a h}$ curve is elaborated on the base of Boltzman distribution [9]. For anisotropic magnetic material it is given as $[10,11]$ :

$$
M_{a h}=M_{s}\left(\frac{\int_{0}^{\pi} \mathrm{e}^{\frac{E(1)+E(2)}{2}} \sin \theta \cos \theta \mathrm{d} \theta}{\int_{0}^{\pi} \mathrm{e}^{\frac{E(1)+E(2)}{2}} \sin \theta \mathrm{d} \theta}\right) \text {, }
$$

where for the uniaxial anisotropy $[10](i=1,2)$ :

$$
E(i)=\frac{H_{e}}{a} \cos \theta-\frac{K_{a n}}{M_{s} \mu_{0} a} \sin ^{2}\left(\phi_{i}\right),
$$

whereas for grain oriented (GO) electrical steel [12] $(i=$ $1,2)$ :

$$
\begin{aligned}
& E(i)=\frac{H_{e}}{a} \cos \theta \\
& -\frac{K_{a n}}{M_{s} \mu_{0} a}\left(\cos ^{2}\left(\phi_{i}\right) \sin ^{2}\left(\phi_{i}\right)+\frac{\sin ^{4}\left(\phi_{i}\right)}{4}\right) .
\end{aligned}
$$

In the above equations $H$ is the magnetizing field strength, $M_{s}$ is saturation magnetization, $K_{a n}$ is the average anisotropy energy density, a quantifies domain wall density, $\alpha$ is inter-domain coupling according to the Bloch model. Moreover, $\phi_{1}=(\psi-\theta)$ and $\phi_{2}=(\psi+\theta)$, where $\psi$ is the angle between the magnetization direction and the easy axis of magnetized material. It should be highlighted, that in the both cases, for isotropic material (where $K_{a n}=0$ ) the equation (2) reduces to the Langevin function:

$$
M_{a h}=M_{s}\left(\operatorname{coth}\left(\frac{H_{e}}{a}\right)-\left(\frac{a}{H_{e}}\right)\right),
$$

as it was proposed in the original model [1]. 
The magnetic hysteresis loop $M(H)$ in the JilesAtherton model is described by the following equation [11]:

$$
\frac{\mathrm{d} M}{\mathrm{~d} H}=\frac{\delta_{M}}{1+c} \frac{M_{a h}-M}{\delta k-\alpha\left(M_{a h}-M\right)}+\frac{c}{1+c} \frac{\mathrm{d} M_{a h}}{\mathrm{~d} H},
$$

where $c \in\langle 0,1\rangle$ describes reversibility of magnetization process and the parameter $k$ quantifies the average energy required to break pining site. Moreover, $\delta=1$ for increase and $\delta=-1$ for decrease of magnetizing field $H$ respectively. Additional parameter $\delta_{M}$ guarantees that incremental susceptibility is always positive, what is physically justified. Parameter $\delta_{M}$ is equal 0 when $M_{a h}-M>0$ and $\delta g-1$, when $M_{a h}-M<0$ and $\delta=+1$. Otherwise $\delta_{M}=1$. Value of the flux density $B$ in the material can be simply calculated considering the magnetic constant $\mu_{0}$ :

$$
B=\mu_{0}(M+H) \text {. }
$$

The above form of differential equation stating the JilesAtherton model was the subject of criticism [13]. On the base of discussion, the alternative form of the model was proposed in 1998 by Venkataraman [14], named as the bulk ferromagnetic hysteresis model:

$$
\frac{\mathrm{d} M}{\mathrm{~d} H}=\frac{\delta k c \frac{\mathrm{d} M_{a h}}{\mathrm{~d} H_{e}}+\delta_{M}\left(M_{a h}-M\right)}{\delta k-\delta_{M} \alpha\left(M_{a h}-M\right)-\delta k c \alpha \frac{\mathrm{d} M_{a h}}{\mathrm{~d} H_{e}}} .
$$

Another problem in both representations of magnetic hysteresis is connected with differential of the function $\frac{\mathrm{d} M_{a h}}{\mathrm{~d} H}$ proposed in the original Jiles-Atherton model as well as in the bulk ferromagnetic hysteresis model. From the physical point of view $\frac{\mathrm{d} M_{a h}}{\mathrm{~d} H_{e}}$ seems to be more physically judged and can be easily accurate estimated by the numerical methods. However, to solve the differential equation (6) in the original Jiles-Atherton model [9] and equation (8) in the bulk ferromagnetic hysteresis model, the chain rule should be used, considering differential of the equation (1):

$$
\frac{\mathrm{d} H_{e}}{\mathrm{~d} H}=1+\alpha \frac{\mathrm{d} M}{\mathrm{~d} H} .
$$

As a result:

$$
\frac{\mathrm{d} M_{a h}}{\mathrm{~d} H}=\frac{\mathrm{d} M_{a h}}{\mathrm{~d} H_{e}} \frac{\mathrm{d} H_{e}}{\mathrm{~d} H}=\frac{\mathrm{d} M_{a h}}{\mathrm{~d} H_{e}}\left(1+\alpha \frac{\mathrm{d} M}{\mathrm{~d} H}\right) .
$$

Considering equation (6) and equation (10) the final form of the Jiles-Atherton model, where one can avoid the differential function, $\frac{\mathrm{d} M_{a h}}{\mathrm{~d} H}$ is given as:

$$
\frac{\mathrm{d} M}{\mathrm{~d} H}=\frac{\delta_{M} \frac{M_{a h}-M}{\delta k-\alpha\left(M_{a h}-M\right)}+c \frac{\mathrm{d} M_{a h}}{\mathrm{~d} H_{e}}}{1+c-c \cdot \alpha \frac{\mathrm{d} M_{a h}}{\mathrm{~d} H_{e}}} .
$$

Due to the quite sophisticated form, equation (11) can be simplified further using 'symbolic toolbox' of OCTAVE. The script for this calculation is presented in the file simplification.m.

\section{Implementation and the test cases}

Analyses presented in the section 2 were implemented as the JAmodel function for OCTAVE/MATLAB. The proposed function script may be called in the following variants:
$B=J A \operatorname{model}(H, M s, a, k, c, a l p h a)$

$B=J A \operatorname{model}(H, a, k, c, M s$, alpha,ModelType,SolverType, AnisoType,Kan,psi,IntType)

where $H$ is the matrix of magnetizing field values (in columns), $B$ is output flux density for $H$, Ms, whereas $a, k, c$, alpha describes model parameters. ModelType enables to choose among Jiles-Atherton model given by the equation (6), Jiles-Atherton model given by the equation (11) and bulk magnetic hysteresis model given by the equation (8). Moreover, SolverType specifies solver for ODE (adaptive or fixed step Runge-Kutta algorithm), Aniso Type determines uniaxial or GO anisotropy, Kan introduces the average anisotropic energy density and $p h i$ is the angle between anisotropy easy axis and the direction of the magnetizing field $H$. IntType determines integration algorithm (trapezoidal approximation or adaptive Gauss-Kronrod approximation).

The parameters of the models were determined by Nelder and Mead simplex algorithm optimisation [8] for two different magnetic materials (isotropic $\mathrm{Mn}-\mathrm{Zn}$ ferrite, GO electrical steel M127-27s), considering three test cases for each material:

Case 1: Jiles-Atherton model, $\mathrm{d} M_{a h} / \mathrm{d} H$,

Case 2: Jiles-Atherton model, $\mathrm{d} M_{a h} / \mathrm{d} H_{e}$,

Case 3: bulk ferromagnetic hysteresis model, $\mathrm{d} M_{a h} / \mathrm{d} H_{e}$

All samples were not subjected to mechanical stresses. Measurements were carried out at the room temperature with magnetizing field frequency $f=0.5 \mathrm{~Hz}$. Direction of easy axis of GO steel was known from production process $(\phi=0)$. The examples of the results of simulation are given in the figure 1a for isotropic and figure 1a GO material, whereas determined parameters for isotropic and anisotropic materials are presented in Tables I and II, respectively.

TABLE I

Parameters obtained for the isotropic material.

\begin{tabular}{c|c|c|c}
\hline \hline Parameter & Case 1 & Case 2 & Case 3 \\
\hline$M_{s}[\mathrm{kA} / \mathrm{m}]$ & 318 & 318 & 318 \\
$a[\mathrm{~A} / \mathrm{m}]$ & 12.6 & 12.6 & 12.6 \\
$k[\mathrm{~A} / \mathrm{m}]$ & 12.4 & 12.5 & 22.9 \\
$c$ & 0.84 & 0.83 & 0.45 \\
$\alpha\left[\times 10^{-8}\right]$ & 11 & 11 & 4.9 \\
\hline$R^{2}$ & 0.9996 & 0.9997 & 0.9997
\end{tabular}

\section{Conclusions}

Presented results indicate that in the case of isotropic magnetic materials, both Jiles-Atherton model as well as bulk magnetic hysteresis model well represents the shape of magnetic hysteresis loops in wide range of amplitudes of magnetizing field. However, for different variants of model, value of parameters $k$ and $c$ vary significantly from 


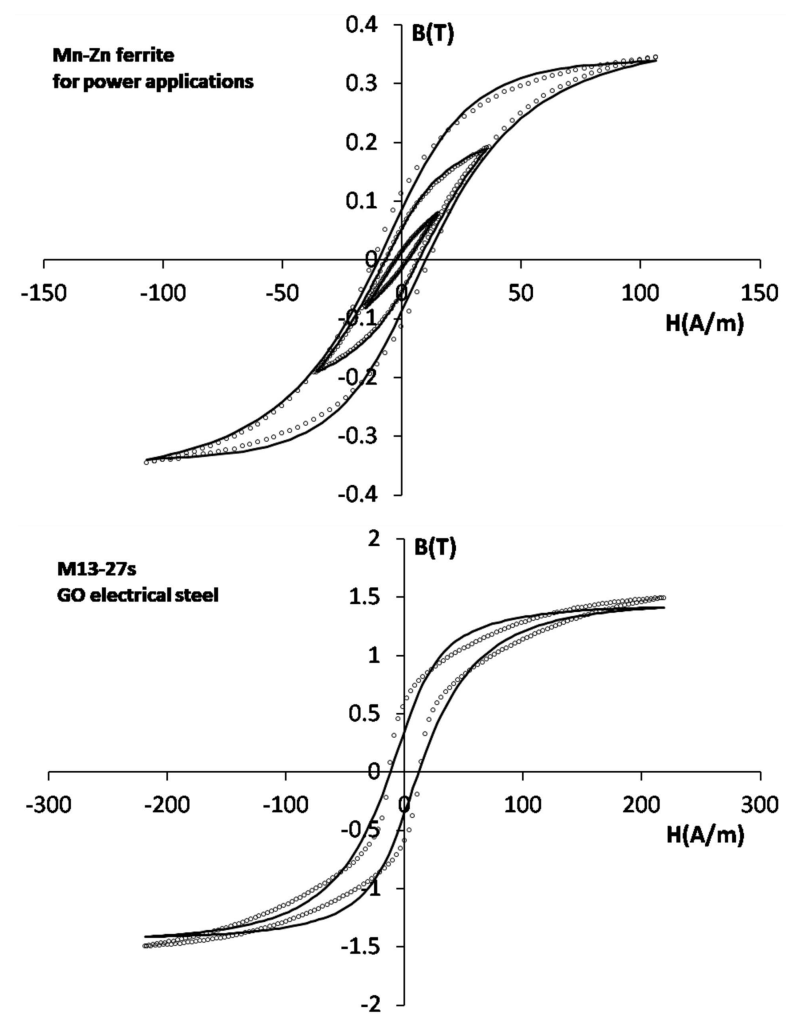

Fig. 1. Results of modelling for bulk magnetic hysteresis model: (top) isotropic $\mathrm{Mn}-\mathrm{Zn}$ ferrite for power applications,(bottom) grain oriented silicon electrical steel M130-27s.

TABLE II

Parameters obtained for the anisotropic material.

\begin{tabular}{c|c|c|c|c|c|c}
\hline \hline \multirow{2}{*}{ Parameter } & \multicolumn{2}{|c|}{ Uniaxial anisotropy } & \multicolumn{2}{c}{ GO type anisptropy } \\
\cline { 2 - 7 } & Case 1 & Case 2 & Case 3 & Case 1 & Case 2 & Case 3 \\
\hline$M_{s}[\mathrm{MA} / \mathrm{m}]$ & 1.26 & 1.23 & 1.21 & 1.26 & 1.22 & 1.21 \\
$a[\mathrm{~A} / \mathrm{m}]$ & 22.5 & 15.1 & 14.3 & 22.6 & 15.1 & 14.3 \\
$k[\mathrm{~A} / \mathrm{m}]$ & 11.7 & 15.1 & 31.2 & 11.7 & 15.1 & 31.2 \\
$c$ & 0.87 & 0.59 & 0.49 & 0.87 & 0.59 & 0.49 \\
$\alpha\left[\times 10^{-6}\right]$ & 16.3 & 1.66 & 1.45 & 16.3 & 1.66 & 1.45 \\
$K_{\text {an }}\left[\mathrm{J} / \mathrm{m}^{3}\right]$ & 13800 & 0.002 & 0.02 & 13800 & 0.002 & 0.02 \\
\hline$R^{2}$ & 0.969 & 0.995 & 0.995 & 0.969 & 0.995 & 0.995
\end{tabular}

values achieved for original model, as a result of different approach to hysteresis modelling.

In the case when magnetic anisotropy is considered in the model, for uniaxial and GO electrical steel, original Jiles-Atherton model (Case 1) didn't reach sufficient convergence during the estimation of model parameters $\left(R^{2}=0.969\right)$. In spite of the fact, that models based on equations (8) and (11) converged, achieved values of average anisotropy energy density $K_{a n}$ seems to be too small from the physical point of view. This effect may be caused by ambiguous values and dependences among Jiles-Atherton model parameters.

On the base of achieved results it should be indicated, that original Jiles-Atherton model and its modifications require further validation in connection with micro-magnetic measurements or specific cases of magnetic materials, as it was carried out for anhysteretic curve previously [11]. Only such validation can confirm proper dependences in Jiles-Atherton model, as commonly used, optimisation-oriented methods of determination of model parameters [5-7] seems to be not reliable enough for anisotropic materials.

\section{References}

[1] D.C. Jiles, D. Atherton, J. Appl. Phys. 55, 2115 (1984).

[2] D. C. Jiles, A. Ramesh, Y. Shi, X. Fang, IEEE Trans. Magn. 33, 3961 (1997).

[3] S. Q. Antonio, M. Pompei, AEIT International Annual Conference, 2015.

[4] K. Chwastek, Math. Comput. Model. Dyn. Syst. 15, 1 (2009).

[5] D. C. Jiles, J. B. Thoelke, M. K. Devine, IEEE Trans. Magn. 28, 1 (1992).

[6] K. Chwastek, J. Szczygłowski, Electrical Review 84, 12 (2008).

[7] R. Biedrzycki, D. Jackiewicz, R. Szewczyk, JAMRIS 4, 63 (2014).

[8] R. Szewczyk, Advances in Intelligent Systems and Computing, Vol. 267, Springer, 2014, p. 275.

[9] D. C. Jiles, D. L. Atherton, J. Magn. Magn. Mater. 61, 48 (1986).

[10] A.Ramesh, D.C. Jiles, J.M. Roderick, IEEE Trans. Magn. 32, 4234 (1996).

[11] R. Szewczyk, Materials, 7, 5109 (2014).

[12] A. P. S. Baghel, S. V. Kulkarni, J. Appl. Phys. 113, 043908 (2013).

[13] S.E. Zirka, Y.I. Moroz, R.G. Harrison, K. Chwastek, J. Appl. Phys. 112, 043916 (2012).

[14] R. Venkataraman, P.S. Krishnaprasad, Proceedings of the 37th IEEE Conference on Decision and Control (1998). 